\title{
SUR LA VARIATION DE L'INDICE DE REICHERT-WOLNY DANS LES BEURRES DE LA RÉPUBLIQUE ARGENTINE
}

\author{
par PAUL LAVENIR \\ Ingénieur agronome I. N. A., Chimiste, \\ Chef du Laboratoire du Ministère national de l'agriculture (Buenos-Aires).
}

Considérations générales.

Dans le courant de l'année 1919 et plus spécialement pendant le second semestre, le commerce d'exportation des beurres argentins jusqu'alors relativement peu important prit subitement un essor considérable et les quantités de beurre exportées, déjà élevées pendant les derniers trimestres de cette année, ne firent qu'augmenter durant les années suivantes, 1920 et 1921, de même que pendant le premier semestre 1922.

En Argentine, les beurres ainsi que tous les autres produits d'origine animal destinés à l'exportation sont soumis à un contrôle officiel exercé par le service d'inspection de police sanitaire dépendant du Ministère national de l'agriculture; à ce contrôle collabore le laboratoire de chimie dudit Ministère, son rôle, dans ce cas, consistant à examiner les échantillons qui lui sont remis par les inspecteurs du service précité, afin de déterminer si les produits desquels ils proviennent répondent aux conditions établies par les règlements adoptés dans le pays, tout en tenant eompte, dans la mesure du possible, des dispositions adoptées par les diverses nations qui importent ces produits.

Pour ce qui concerne le beurre, on sait que les dispositions réglementant son commerce sont en général assezi ncomplètes. La plupart indiquent un maximum de teneur en eau, un minimum de matières grasses, elles fixent en outre la quantité de sel que les beurres pourront contenir ainsi que la nature des eolorants tolérés ; quelques pays admettent dans les beurres une certaine dose d'acide boriqùe ou de borax à exclusion de tout autre antiseptique. Jusquelà aucune difficulté sérieuse ne se présente au chimiste chargé de l'examen des beurres, mais il en est tout autrement lorsqu'il doit se prononcer sur leur pureté, c'est-à-dire sur la question de savoir s'ils sont exempts de matières grasses étrangères au lait, comme l'exigent toutes les réglementations relatives à la vente de ce produit.

Parmi les constantes physico-chimiques qui peuvent éclairer l'expert sur la pureté des beurres, une surtout a une importance considérable, c'est celle qui a pour but d'évaluer leur teneur en acides volatils, connue sous le nom d'indice de Reichert. Nombreuses sont les modifications apportées à la méthode primitive de 
détermination de cet indice, mais, en raison de l'obligation d'abréger le plus possible le travail des chimistes et du nombre élevé d'échantillons à examiner, la méthode que nous avons adoptée est celle relativement simple et rapide de Reichent-Wozny; elle consiste, comme on le sait, à évaluer en centimètres cubes de potasse $\mathrm{N} / 10$ la partie des acides volatils solubles provenant de $5 \mathrm{gr}$. de matières grasses du beurre. Cette méthode adoptée par de nombreux chimistes fournit, lorsqu'elle est pratiquée par un personnel expérimenté, des résultats suffisamment concordants, bien que conventionnels et, comme tels, surtout comparables entre eux. Malgré la simplicité relative de cette méthode il n'a pas été possible de l'appliquer à tous les échantillons qui nous ont été remis pendant les années 1920 et 1921 , c'est ainsi que sur 2.645 échantillons l'indice R.-W. n'a été déterminé que sur 1.077, c'est-à-dire sur $40 \%$ du total; cependant si l'on considère que chaque échantillon corrrespond à un lot important de beurre embarqué dont il représente la moyenne, on peut admettre que les résultats obtenus sur $40 \%$ de ces lots, pendant les deux années consécutives, donnent une idée assez exacte de la caractéristique des beurres de production argentine, en ce qui a trait à leur richesse en acides volatils solubles et par que conséquent ils ne manquent pas d'intérêt.

Dans les pays européens en général, surtout pour ceux dont l'agriculture est avancée, les caractères des beurres de chaque région productrice de lait sont connus ; de nombreuses études et des statistiques sérieuses ont permis de les établir, tout au moins d'une facon suffisante pour pouvoir les utiliser avec fruit comme base de comparaison dans des cas douteux. Il n'en est pas de même en Argentine; le développement de l'industrie beurrière dans ce pays est relativement récent, les régions qui produisent les crèmes traitées ensuite dans des établissements souvent d'une grande importance, oceupent de vastes espaces, elles sont, de plus, éloignées les unes des autres et, par conséquent, soumises à des conditions de sol et de climat bien différentes. Comme chaque établissement beurrier, suivant les puissances des moyens mécaniques dont il dispose et afin de s'assurer pendant toute l'année une quantité suffisante de matière première, reçoit les crèmes à la fois de plusieurs régions, il s'en suit que les résultats obtenus dans l'examen des beurres provenant de ces fabriques ne sont pas applicables aux beurres de telle ou telle région, mais bien à des mélanges provenant d'un certain nombre de celles-ci, dans lesquelles les conditions de climat et d'alimentation des animaux sont loin d'être semblables. Cette circonstance a pour effet d'atténuer les variations que peuvent présenter les constantes physico-chimiques des beurres; malgré cela les écarts que l'on con- 
state dans l'indice de R.-W. des beurres d'exportation, indice duquel nous nous occuperons exclusivement dans cette étude sont, comme nous le verrons plus loin, assez considérables et dans certains cas, sa valeur est si inférieure au minimum généralement admis pour les beurres normaux qu'on pourrait être conduit à mettre en doute leur état de pureté. Les écarts que nous signalons ne représentent cependant pas les variations extrêmes de l'indice R.-W. des beurres Argentins dans l'état actuel de l'industrie laitière; pour les établir, il faudrait effectuer une étude de ce produit par région, en entendant comme telle des espaces plus ou moins vastes n'offrant pas de différences importantes au point de vut climatérique et agrologique. Néanmoins, les résultats que nous exposons dans cette étude sont très intéressants puisqu'ils correspondent aux beurres d'exportation que l'industrie beurrière du pays fabrique en quantités énormes; ce sont en définitive les beurres que les experts des pays importateurs auront à juger et il est donc très utile de leur faire connaître les particularités qu'offrent ces beurres, en ce qui concerne leur richesse en acides volatils. Ce fait est d'autant plus important que certains pays, l'Italie en particulier, fixent officiellement pour les beurres normaux un indice R.-W. de 26,0 et que l'on peut penser que d'autres suivront cet exemple.

\section{Observations relatives à l'indice de Reichert-Wolny.}

Les tableaux I et II se rapportent à l'année 1920, les tableaux III et IV, à l'année 1921. Pour chaque mois de l'année, on trouvera dans les tableaux I et III les maxima, minima et moyennes de l'indice R.-W. ; dans les tableaux II et IV, les échantillons ont été classés par mois suivant l'ordre croissant de l'indice de R.-W., variant d'une unité.

\begin{tabular}{|c|c|c|c|c|c|c|}
\hline & & & TABLI & $\begin{array}{l}\text { I } \\
\text { Indice } \\
\text { chert-I }\end{array}$ & & Nombre \\
\hline & & & Moy. & Min. & Max. & d'échantillons \\
\hline Janvier . &. & . & 27,7 & 25,4 & 29,8 & 21 \\
\hline Février. & .. & . & 25,8 & 24,4 & 28,8 & 27 \\
\hline Mars . . & . . & . & 26,4 & 24,3 & 29,8 & 50 \\
\hline Avril . . & . . & . . & 26,7 & 24,1 & 30,0 & 80 \\
\hline Mai . . & . & . . & 26,6 & 23,5 & 29,9 & 114 \\
\hline Juin , , . & . . & . . & 25,6 & 24,2 & 28,5 & 65 \\
\hline Juillet . & . . & . . & 25,4 & 22,9 & 29,0 & 43 \\
\hline Août . . & . . & . & 25,1 & 22,0 & 29,2 & 30 \\
\hline Septembre & . . & 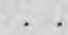 & 27,0 & 24,5 & 30,0 & 47 \\
\hline Octobre. & . . & . & 29,1 & 25,4 & 30,9 & 74 \\
\hline Novembre & . . & . & 29,1 & 26,8 & 31,2 & 103 \\
\hline Décembre & . . & . . & 29,2 & 27,0 & 32,0 & 72 \\
\hline & & & 26,6 & 22,0 & 32,0 & 726 \\
\hline
\end{tabular}




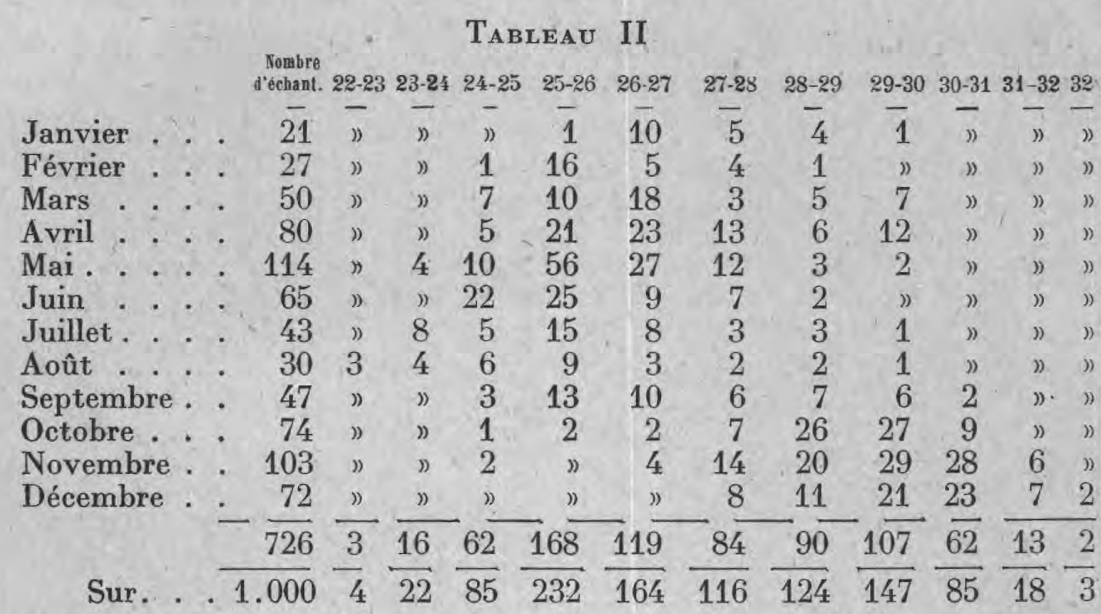

L'examen de ces résultats démontre tout d'abord que, pendant les deux années que comprend cette étude, la valeur de l'indice R.-W. a varié dans les limites suivantes :

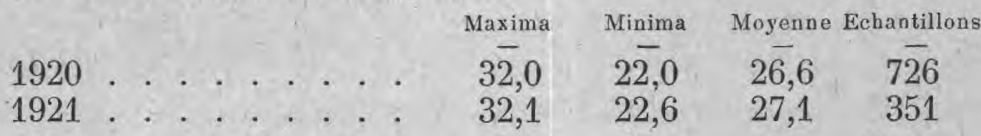

Ces résultats sont peu différents d'une année à l'autre et l'on peut dire que, sur le total d'échantillons, la.moyenne de l'indice R.-W. est de 26,7 , comprise entre 22,0 et 32,1 .

\section{TaBLEaU III}

\begin{tabular}{|c|c|c|c|c|c|c|}
\hline & & & $\begin{array}{c}\text { Nombre d'ée } \\
\text { tillous }\end{array}$ & Moyenne & Minimum & Maximu \\
\hline Janvier & . & & 39 & 28,3 & $2 \overline{6}, 1$ & 30,0 \\
\hline Février & 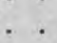 & . & 13 & 25,2 & 24,3 & 27,4 \\
\hline Mars. & . & 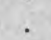 & 14 & 25,8 & 24,0 & 27,9 \\
\hline Avril. & . & . & 41 & 25,3 & 23,8 & 28,7 \\
\hline Mai . & . & * & 20 & 25,1 & 23,6 & 26,5 \\
\hline Juin . & . & . & 14 & 25,0 & 22,6 & 28,9 \\
\hline Juillet . & . . & . & 14 & 25,2 & 23,2 & 28,4 \\
\hline Août & . . & . & 25 & 25,2 & 22,6 & 28,9 \\
\hline Septembre & & . & 30 & 26,4 & 23,5 & 29,1 \\
\hline Octobre. & . . & . & 37 & 27,4 & 24,7 & 30,6 \\
\hline Novembre & . . & . & 75 & 29,2 & 25,0 & 32,1 \\
\hline écembre & & & 29 & 27,6 & 25,5 & 29,7 \\
\hline & & & 351 & 27,1 & 22,6 & 32,1 \\
\hline
\end{tabular}

Les tableaux II et IV démontrent comment, dans le courant de chaque mois, varie l'indice de R.-W; mais, si l'on considère l'année entière, on voit que pour 1920 la majeure partie des échantillons, soit $78 \%$ au total, ont l'indice de R.-W. compris entre 25,0 et 30,0 ; 
$89 \%$ de ces échantillons ont cet indice supérieur à 25,0 et $97 \%$ l'ont au-dessus de 24,0. Pour 1921 ces proportions sont à peu près les mêmes, elles peuvent donc, sans erreur appréciable, être admises pour les deux années comme résultats de l'examen de 1.077 échantillons, parvenant de toutes les régions laitières du pays.

\section{TABLEAU IV}

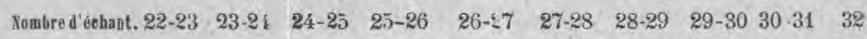

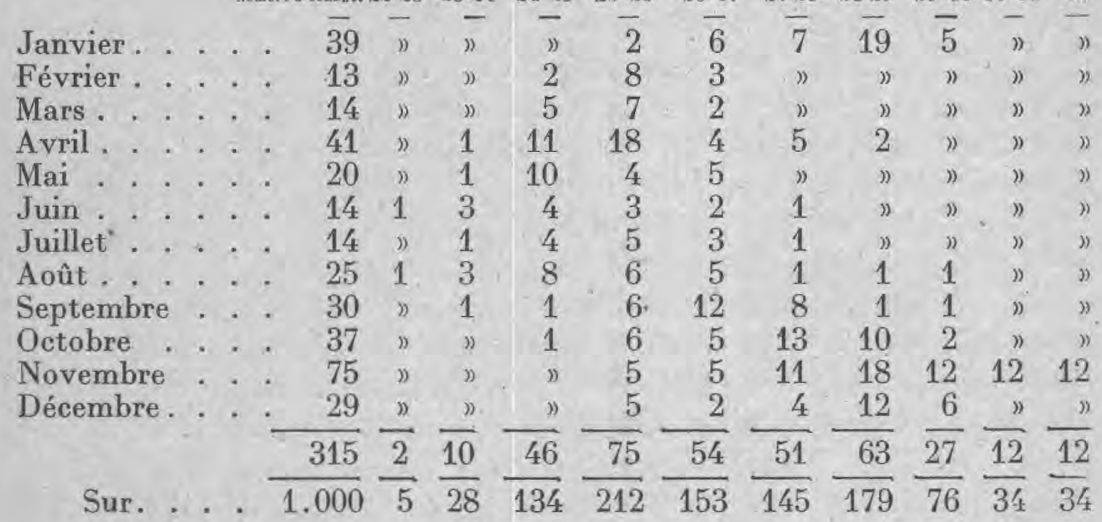

Il est certain que, comme l'indiquent ces résultats, la valeur de l'indice R.-W. des beurres argentins varie entre de grandes limites et si l'on envisage les dispositions prises par l'Italie, on voit que seulement $65,7 \%$ des beurres exportés en 1920 et $62,1 \%$ de ceux expédiés en 1922, seraient compris dans la limite adoptée par ce pays pour l'indice de R.-W.; les autres pourraient, en conséquence, être classés dans les anormaux et même considérés comme suspects ceux de ces beurres dont l'indice est au-dessous de 24,0, et qui représentent environ $6 \%$ des lots.

A quoi attribuer ces variations? Tout d'abord on doit écarter la question de fraude par addition de graisses étrangères au lait; la surveillance exercée dans les établissements, même par le service d'inspection du Ministère de l'agriculture, est déjà une garantie à ce sujet, mais, en ne considérant que les deux années 1920-1921, le prix relativement élevé à cette époque des graisses pouvant être employées dans le but de fraudes, comparé à celui du beurre, ne permet guère de réaliser des bénéfices intéressants en Argentine, sauf le cas de recourir à une adultération grossière par le poureentage élevé de graisses ajoutées aux beurres, opération qui, du reste, serait alors facile de déceler. Le fait est que les nombreuses investigations que nous avons pratiquées tant dans l'industrie qu'au laboratoire nous ont conduit à rejeter l'hypothèse de la fraude; nous sommes done en condition d'affirmer que les beurres que nous avons 
examinés en 1920-1921 et nous ajouterons, de même que ceux des années suivantes, étaient exempts de graisses étrangères au lait. Sur ce point, les résultats eonsignés dans les tableaux II et IV nous éclairent, ils font en effet ressortir les causes de variation de l'indice de R.-W.; leur examen nous montre, en effet, que les plus faibles indices correspondent aux mois de mai, juin, juillet et août qui sont les plus froids en Argentine et que, par contre, les indices les plus élevés se trouvent dans les mois d'octobre, novembre, décembre et janvier qui sont les plus chauds. Il n'est donc pas douteux que les conditions climatériques influent sur la constitution des beurres; tout d'abord, il est bon de tenir compte du fait que les vaches laitières sont maintenues constamment au pâturage, c'est-à-dire au dehors, soumises à toutes les intempéries sans aucun abri. Nous ne voulons eependant pas affirmer que la température, les précipitations atmosphériques et autres conditions climatériques agissent directement sur le taux des acides volatils solubles des beurres, d'une façon aussi marquée que celle constatée, mais leur influence est considérable en ce qui concerne l'alimentation des vaches laitières; il suffit en effet d'une sécheresse prolongée et de quelques jours de gelée pour qu'en hiver les animaux exclusivement au pâturage, ne recevant aucun complément de nourriture, se trouvent soumis à un régime alimentaire de plus en plus défectueux et insuffisant; ces animaux dont l'état de maigreur s'accentue tous les jours fournissent alors un beurre contenant des proportions d'acides volatils de plus en plus réduites; e'est ainsi que nous avons trouvé des indices de R.-W. de 20,0 dans des produits provenant de régions qui, pendant les saisons des bons pâturages, fournissent des beurres dont cet indice atteint 28,0 et 30,0 . C'est done surtout à une alimentation insuffisante que l'on doit attribuer nous ne dirons pas exclusivement, mais principalement, les variations de l'indice de R.-W. dans les beurres que nous avons examinés, insuffisance qui se fait sentir surtout l'hiver en raison de la sécheresse et des gelées qui souvent agissent simultanément. Le manque de nourriture peut également se faire sentir, bien qu'à un degré moindre, dans la saison chaude, ehaque fois que des conditions elimatériques défavorables à la végétation se présentent et persistent pendant un temps suffisamment prolongé. Si, pour ce motif, les animaux maigrissent, on voit alors baisser progressivement l'indice de R.-W. des heurres qu'ils produisent.

D'autres circonstances et, parmi elles, la fièvre aphteuse généralisée, provoqueront peut-être des changements importants dans la constitution des beurres; mais nous n'avons pas encore étudié le cas de la fièvre aphteuse. 
Quelques exemples démontreront le bien fondé de ces observations.

Des échantillons de beurres prélevés du 10 au 16 juin dans une fabrique importante, située dans la capitale, à proximité du Laboratoire, avaient un indice de R.-W. de 27,3 et 25,1 , mais par la suite, cet indice baissa progressivement, à tel point que du 15 au 23 juillet il n'était plus qu'à $24,3-24,0$. II convient de remarquer que les beurres analysés ont été préparés en présence d'un employé du Laboratoire qui assista à toutes les opérations que comporte la fabrication, depuis l'arrivée des crèmes et leur mélange, jusqu'au sortir des beurres de la baratte. Ces crèmes provenaient en grande partie de la région nord-est du territoire de la Pampa et de la région voisine de la province de Buenos-Aires, régions exposées à la sécheresse et aux gelées. A la même époque, des erèmes d'une région plus centrale de la province de Buenos-Aires, moins éprouvée que les précédentes, donnaient des beurres dont l'indice R.-W. était de 26,3-26,0.

Un autre exemple très démonstratif est celui-ci : Dans une région dans laquelle, pour les raisons indiquées, les vaches manquaient de nourriture et donnaient vers la fin d'août des beurres avec un indice R.-W. de $22,4-20,3$, il se trouva que quelques exploitations distribuaient à leurs animaux un complément de nourriture consistant en fourrages ensilés; les crèmes provenant de ces établissements à la même époque que les antérieures donnaient des beurres dont l'indice de R.-W. était de 26,3; du reste cet indice baissa plus tard, les réserves de fourrages ensilés s'étant épuisées avant que les pâturages se soient suffisamment développés pour maintenir les animaux en bon état.

Autres exemples : Deux échantillons de beurre fabriqués fin août dans un important établissement recevant ses crèmes de l'ouest de la province de Buenos-Aires donnaient un indice R.-W. de 24, 2-22,8. Deux autres échantillons préparés au Laboratoire avec des crèmes de la même région, recueillies sur place par un emploýé du Laboratoire, avaient un indice R.-W. encore plus bas, puisqu'il n'atteignait que $21,1-21,0$.

Six échantillons obtenus de la même façon que les deux précédents, c'est-à-dire préparés au Laboratoire avec des crèmes séparées au lieu même de production, en présence d'un employé du Laboratoire, donnèrent les indices de R.-W. suivants : 24,3 - 23,6 - 23,5 - 22,3 - 21,3 et 20,8 ; tandis qu'un autre beurre préparé dans les mêmes conditions et en même temps que les six précédents avec de la erème provenant d'une région semée de luzerne et dans laquelle les vaches laitières, bien nourries, s'étaient maintenues dans un état normal, avait un indice R.-W. de 29,8 .

Nous pourrions multiplier les exemples, mais nous croyons inutile 
d'insister ; il n'est pas douteux que le régime alimentaire auquel se trouvent soumises les vaches laitiêres en Argentine est la cause principale des variations que l'on constate dans la teneur des beurres en acides volatils solubles, variations desquelles il faut tenir compte lorsqu'il s'agit d'apprécier la pureté des beurres que ce pays produit. En se rapportant aux tableaux I, II, III, IV, qui correspondent aux beurres du commerce, on voit que si pendant les mois à végétation en général abondante, rares sont les beurres ayant un indice R.-W. inférieur à 25,0 , il n'en est pas de même pour les beurres d'hiver.

Actuellement le Gouvernement s'occupe activement de l'amélioration de l'industrie laitière, la question de l'alimentation des vaches laitières est à l'ordre du jour et une propagande active est faite dans le but d'amener les producteurs, de crème à préparer des réserves alimentaires, destinées à compenser l'insuffisance des pâturages à certaines époques de l'année. D'autre part, l'industrie s'efforce de n'expédier à l'étranger que des beurres à indice R.-W. normal ou considéré comme tel, c'est-à-dire non inférieur à 25,0 et autant que possible non au-dessous de 26,0 .

Ce qui précède est un extrait sous une forme résumée d'une étude publiée par le Ministère de l'agriculture en 1923, sous le titre de Contribucion al estudio de las mantecas Argentinas. Nous croyons intéressant d'y ajouter d'assez nombreux résultats obtenus depuis au Laboratoire dudit Ministère et que nous avons groupés dans deux tableaux, V et VI, identiques, quand à leur disposition, aux précédents. Ces résultats se rapportent à l'année 1923; nous y avons joint également ceux obtenus dans le courant de janvier et de février de l'année 1924. Le nombre d'échantillons analysés pendant ces quatorze mois s'élève à 604 et la valeur moyenne de l'indice R.-W.,

\begin{tabular}{|c|c|c|c|c|c|c|c|c|}
\hline & & & & & Echantillons & Moyenne & Minimum & Maximum \\
\hline Janvier & 1923 & & & & 43 & $2 \overline{7}, 8$ & $2 \overline{5}, 0$ & $3 \overline{0}, 9$ \\
\hline Février & - & . & $\therefore$ & & 27 & 25,5 & 24,2 & 27,8 \\
\hline Mars & - & . & 2. & & 26 & 25,8 & 23,0 & 28,0 \\
\hline Avril & - & . . & . . & & 30 & 25,3 & 24,0 & 27,4 \\
\hline Mai & - & . . & . . & & 13 & 25,4 & 24,1 & 27,1 \\
\hline Juin & - & 1. & $\therefore$. & . & 34 & 25,2 & 23,5 & 27,1 \\
\hline Juillet & - & . & 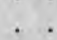 & & 74 & 24,6 & 23,4 & 28,1 \\
\hline Août & - & . & • & & 63 & 25,1 & $22 ; 3$ & 28,7 \\
\hline Septem. & - & . . & . . & & 53 & 27,5 & 24,1 & 29,5 \\
\hline Octobre & - & . . & . . & " & 66 & 30,1 & 25,5 & 32,1 \\
\hline Novem. & - & . . & . . & & 47 & 31,3 & 28,7 & 32,9 \\
\hline Décemb. & - & $\therefore$ & . . & & 12 & 30,8 & 29,1 & 33,1 \\
\hline Janvier & 1924 & . & . . & , & 46 & 30,7 & 28,5 & 32,8 \\
\hline Février & - & . & . . & & 60 & 28,7 & 25,3 & 32,7 \\
\hline & & & & & $\overline{604}$ & $\overline{26,7}$ & 22,3 & 33,1 \\
\hline
\end{tabular}


obtenue pendant cette période, est de 26,7 comprise entre 22,3 et 33,1 (tableau V). Ces chiffres ne diffèrent pas sensiblement de ceux trouvés pour les années 1920-1921, mais ils viennent confirmer les variations que subit la teneur en acides gras volatils des beurres argentins et contribuent à démontrer l'influence des conditions climatériques et surtout de l'alimentation sur l'indice de R.-W. L'examen du tableau VI est très suggestif à cet égard; en effet; il

\section{TABleau VI}

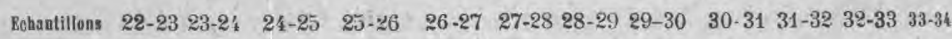

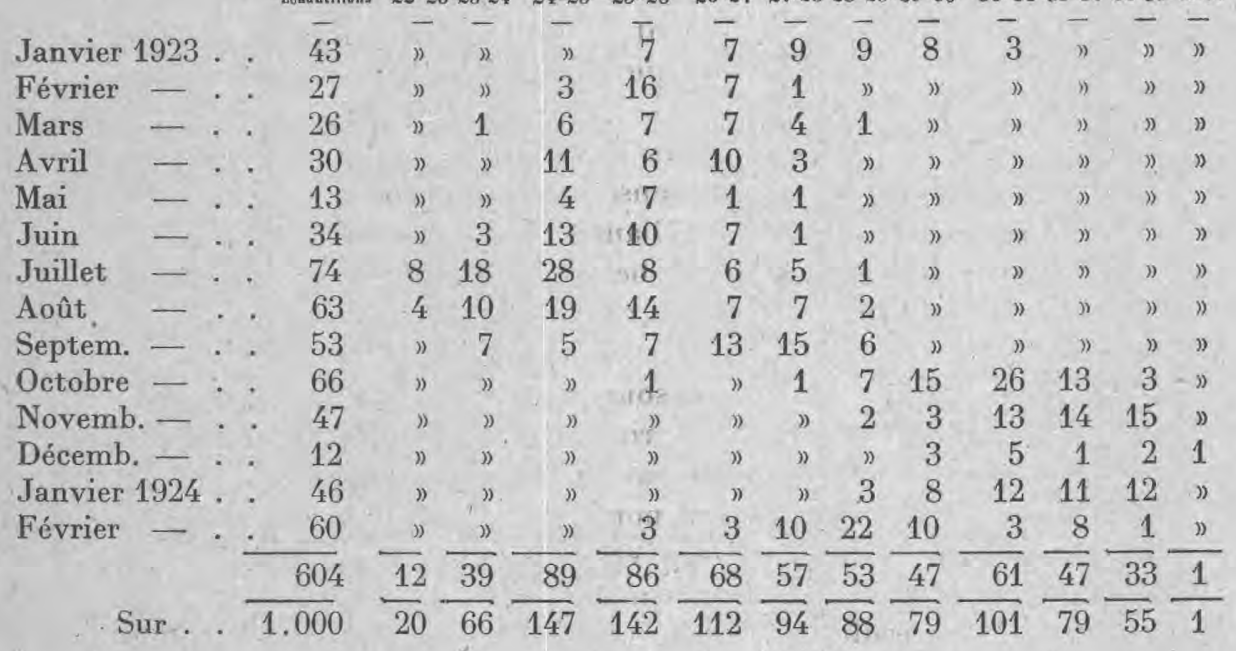

indique, d'une façon très nette, que pendant les mois les plus froids, juin, juillet, août et septembre, la valeur de l'indice R.-W. peut baisser jusqu'à $22,0-23,0$ et ne dépasse pas $28,0-29,0$, tandis que pendant la saison chaude, octobre, novembre, décembre, janvier et février, rares sont les cas où cette valeur est inférieure à $27,0-28,0$, tandis qu'elle atteint jusqu'à 33,0 .

Ces différences apparaissent plus clairement sur ce tableau que sur les tableaux II et IV à cause de l'hiver de 1923 qui fut particulièrement rigoureux, surtout dans la région ouest de la province de Buenos-Aires et dans la voisine du territoire de la Pampa, régions qui fournissent à l'industrie beurrière des quantités considérables de erème; par contre, pendant les mois chauds, les conditions de température et d'humidité se sont montrées très favorables à la prod tion herbacée, les pâturages furent donc abondants pendant ce záriode de l'année tandis qu'en hiver les animaux manquèrent d ncurriture par suite de sécheresse prolongée et de gelées succes- 